\title{
Noise-reduction effects of oven magnetron with cathode shield on high-voltage input side
}

$\operatorname{AUTHOR}(S)$ :

Mitani, T; Shinohara, N; Matsumoto, H; Aiga, M; Kuwahara, N; Ishii, T

\section{CITATION:}

Mitani, T ...[et al]. Noise-reduction effects of oven magnetron with cathode shield on highvoltage input side. IEEE TRANSACTIONS ON ELECTRON DEVICES 2006, 53(8): 1929-1936

\section{ISSUE DATE:}

2006-08

URL:

http://hdl.handle.net/2433/39988

\section{RIGHT:}

(c)2006 IEEE. Personal use of this material is permitted. However, permission to reprint/republish this material for advertising or promotional purposes or for creating new collective works for resale or redistribution to servers or lists, or to reuse any copyrighted component of this work in other works must be obtained from the IEEE. 


\title{
Noise-Reduction Effects of Oven Magnetron With Cathode Shield on High-Voltage Input Side
}

\author{
Tomohiko Mitani, Member, IEEE, Naoki Shinohara, Member, IEEE, Hiroshi Matsumoto, Fellow, IEEE, \\ Masayuki Aiga, Nagisa Kuwahara, and Takeshi Ishii
}

\begin{abstract}
A magnetron with a metallic cathode shield on the high-voltage (HV) input side is newly designed for the purpose of reducing the spurious noise generated from an oven magnetron. In this paper, it was experimentally found that the newly designed magnetron suppressed the sideband noise around the carrier frequency up to $10 \mathrm{~dB}$, compared to the conventional magnetron. Moreover, both the spurious noise in the high frequency bands (4-14 GHz) and the line noise in the low frequency bands ( $\sim 1 \mathrm{GHz}$ ) from the newly designed magnetron were reduced up to $30 \mathrm{~dB}$, compared to the conventional one. It was also found that a cathode shield attached to only the $\mathrm{HV}$ input side was more effective than the cathode shields attached to both the HV input side and the RF output side, with respect to the noise reduction. The thermionic emission from the cathode filament and the motions of the electrons in a magnetron are discussed in investigating the noise-reduction mechanisms.
\end{abstract}

Index Terms-Cathodes, magnetrons, noise.

\section{INTRODUCTION}

A MAGNETRON is a well-known crossed-field device commonly used for a microwave heating source of an oven. It is also expected as one of the candidates for a microwave source of the "wireless power transmission (WPT)" technology, because of its high dc-RF conversion efficiency, low weight/power ratio and low production cost. A WPT system is especially suitable for transmitting electricity to places where electric wires are difficult or impossible to install. Future applications of a WPT system might be for power transmission to the isolated islands, between space satellites, or from satellites to the Earth, the so-called "solar power satellite (SPS)" concept [1].

A free-running magnetron has a wide bandwidth and spurious noise in the various frequency bands. For the microwave ovens, additional circuits and elements are accordingly necessary to filter out the line noise below $1 \mathrm{GHz}$, to avoid the interference with radio, TV, and the other electric systems. In the WPT case, the spurious noise from a magnetron will interfere in the other communication systems when they are radiated from the transmitters of a WPT system. Therefore, it

Manuscript received January 18, 2006; revised May 23, 2006. The review of this paper was arranged by Editor W. Menninger.

T. Mitani and N. Shinohara are with the Research Institute for Sustainable Humanosphere (RISH), Kyoto University, Uji 611-0011, Japan (e-mail: mitani@ rish.kyoto-u.ac.jp).

H. Matsumoto was with the RISH until March 2006. He is now with Kyoto University, Uji 611-0011, Japan.

M. Aiga, N. Kuwahara, and T. Ishii are with the Panasonic Semiconductor Discrete Devices Company, Ltd., Utsunomiya 321-0905, Japan.

Digital Object Identifier 10.1109/TED.2006.878238 is indispensable to employ measures against the spurious noise generated from a magnetron.

For the WPT use, the spurious noise is effectively suppressed when a magnetron is operated by a dc-stabilized power supply and the filament current is turned off during the operation. Some experimental researches have shown that this operating method works exceedingly well not only in reducing the bandwidth up to $60 \mathrm{~dB}$ [2], [3] but also in suppressing the high-frequency $(4-10 \mathrm{GHz})$ spurious noise up to $50 \mathrm{~dB}$ and low-frequency $(\sim 1 \mathrm{GHz})$ line noise up to $40 \mathrm{~dB}$ [4]. These achievements contributed to the development of some types of phase-controlled magnetrons (PCM) [5]-[7] and a phase-andamplitude-controlled magnetron (PACM) [8].

For microwave oven use, it has been experimentally demonstrated that a magnetron with an ion-drain electrode effectively reduces the line noise below $1 \mathrm{GHz}$, caused by the trapped ions in the vicinity of the cathode [9]. Alternatively, new techniques called "magnetic priming" [10], [11] or "electric priming" [12] may serve as effective measures against the reduction of the startup noise by hastening the $\pi$-mode selection.

The objectives of this paper are to reduce the spurious noise of an oven magnetron and to develop a low-noise magnetron for oven use. The low-noise magnetron leads to the reduction of the production cost by removing a feedthrough capacitor and choke coils from a conventional oven magnetron. In this paper, we have newly designed a magnetron with a cylindrical metallic cathode shield on the high-voltage (HV) input side of the filament. With regard to the cathode shield, Kohsaka et al. [13] have suggested a magnetron with cathode shields on both the RF output side and the HV input side, in order to prevent the electron emission from the end portions of the cathode.

From the experimental results, we found that the newly designed magnetron suppressed the sideband noise around the carrier frequency up to $10 \mathrm{~dB}$, compared to the conventional magnetron. Moreover, both the spurious noise in the high frequency bands $(4-14 \mathrm{GHz})$ and the line noise in the low frequency bands $(\sim 1 \mathrm{GHz})$ from the newly designed magnetron were reduced up to $30 \mathrm{~dB}$, compared to the conventional one. We also found out that a cathode shield attached to only the HV input side was more effective than the cathode shields attached to both the HV input side and the RF output side, as suggested by Kohsaka et al. [13], with respect to the noise reduction.

\section{Configurations of Magnetrons}

Fig. 1 shows the inside schematics of a conventional magnetron Panasonic 2M210M1F1 [Fig. 1(a)], a magnetron with 


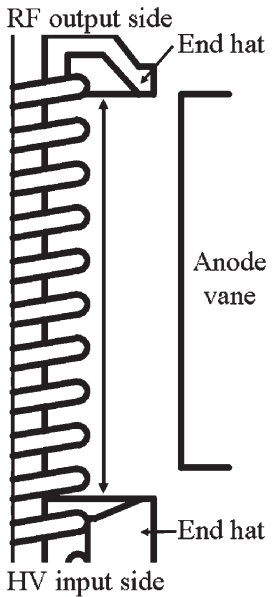

(a)

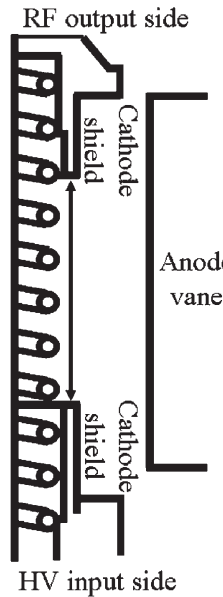

(b)

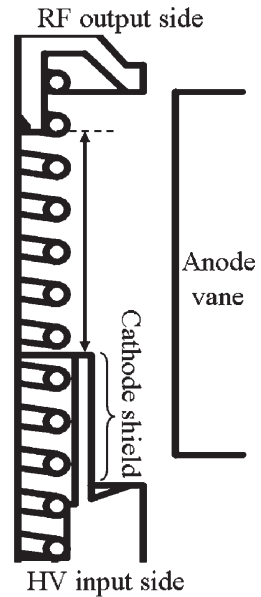

(c)
Fig. 1. Inside schematics of (a) conventional magnetron, (b) magnetron with cathode shields on both sides, and (c) magnetron with a cathode shield on the $\mathrm{HV}$ input side. The effective emission area of each magnetron is shown by a two-headed arrow.

the cathode shields on both sides [Fig. 1(b)], and a magnetron with a cathode shield only on the HV input side [Fig. 1(c)]. The structure of the magnetron (b) is similar to the magnetron depicted by Kohsaka et al. [13], and the metallic cathode shields are directly connected with both end hats in order to prevent the electron emission. The structure of the magnetron (c) is newly designed, and the metallic cathode shield is directly connected only with the end hat on the HV input side.

The effective emission area, where the thermal electrons are expected to be emitted, of each magnetron is shown by a twoheaded arrow in Fig. 1. It is between the cathode shields of the magnetron (b) and between the cathode shield and the brazing point to the end hat of the magnetron (c). The effective emission area of the magnetron (b) is designed to be the same as that of the magnetron (c) and smaller than the conventional magnetron (a). In terms of the other parameters such as the radii of both the anode and the cathode, the anode shape, the rated anode voltage, the external magnetic field, etc., there is no difference among the three types of the magnetrons.

\section{MeAsurements of The SPURious Noise Generated From the Magnetrons}

\section{A. Configurations of the Measurements}

The measurement system of the spurious noise generated from a magnetron is shown in Fig. 2. A magnetron is operated by a half-wave voltage doubler, which is commonly used as a power supply of a microwave oven. The anode current and the filament voltage are fixed at $400 \mathrm{~mA}$ and $3.3 \mathrm{~V}$, respectively, for all the magnetrons. The measurements are conducted in the condition that the feedthrough capacitor and the choke coils attached to the magnetron are removed, in order to detect all the intrinsic spurious noise generated from the magnetron.

The measuring range of the spectrum is divided into three bands: $2.35-2.55 \mathrm{GHz}$ for the fundamental, 4-14 GHz for the high-frequency spurious noise, and $100 \mathrm{kHz}-1 \mathrm{GHz}$ for the low-frequency line noise. In the fundamental frequency bands

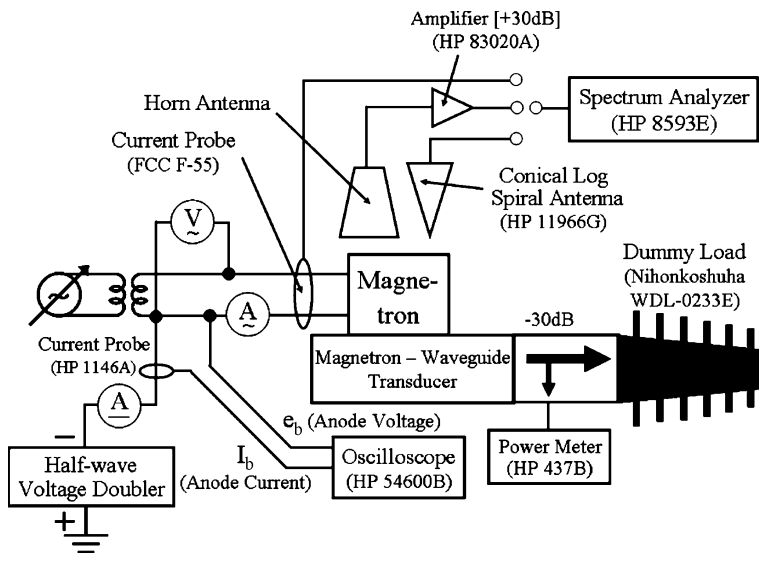

Fig. 2. Schematics of the measurement system. $e_{b}$ : Anode voltage. $i_{b}$ and $I_{b}$ : Anode currents. $V_{f}$ : Filament voltage. $I_{f}$ : Filament current.

TABLE I

SETtings OF THE SPECTRUM ANALYZER. RBW: RESOLUTION BANDWIDTH. VBW: VIDEO BANDWIDTH

\begin{tabular}{|c||c|c}
\hline Frequency span & RBW & VBW \\
\hline \hline $2.35 \mathrm{GHz}-2.55 \mathrm{GHz}$ & $10 \mathrm{kHz}$ & $10 \mathrm{kHz}$ \\
\hline $4 \mathrm{GHz}-14 \mathrm{GHz}$ & $1 \mathrm{MHz}$ & $10 \mathrm{kHz}$ \\
\hline $100 \mathrm{kHz}-1 \mathrm{GHz}$ & $100 \mathrm{kHz}$ & $10 \mathrm{kHz}$ \\
\hline
\end{tabular}

and the high frequency bands, the leakage electromagnetic waves are measured with a spiral antenna and a horn antenna, respectively. Although the absolute value of the spectral output cannot be derived, the measurement system shown in Fig. 2 is adopted, because the main objective of the present research is the relative comparisons of the magnetron noise with each other. In the low frequency bands, the line noise on the cables between the magnetron and the half-wave voltage doubler is measured with a current probe.

The spectrum in each frequency band is measured with a spectrum analyzer. In the present experiment, the maximum value of the spectrum is held on the spectrum analyzer during a period of $1 \mathrm{~min}$ when the magnetron and the waveguide are thermally stable. Thus, the spectrum analyzer displays the envelope of the maximum levels of the spectra generated from the magnetron. The settings of the resolution bandwidth and the video bandwidth of the spectrum analyzer are put in Table I.

\section{B. Experimental Results}

The experimental results of the fundamental spectrum, highfrequency spurious noise, and low-frequency line noise are shown in Fig. 3. The spectra of the conventional magnetron, the magnetron with the cathode shields on both the filament ends, and the magnetron with a cathode shield on the HV input side are plotted in Fig. 3(a)-(c), respectively. The normal-rated filament voltage of $3.3 \mathrm{~V}$ remains applied, and it is not cut back or turned off during the measurements.

With regard to the fundamental spectrum, the sideband noise below $2.43 \mathrm{GHz}$ is less than the noise floor of the spectrum analyzer when the cathode shields are attached to a magnetron. Hence, the cathode shields work well for the sideband noise in the fundamental frequency bands. Moreover, the sideband noise from 2.43 to $2.45 \mathrm{GHz}$ of the magnetron (c) is less than 

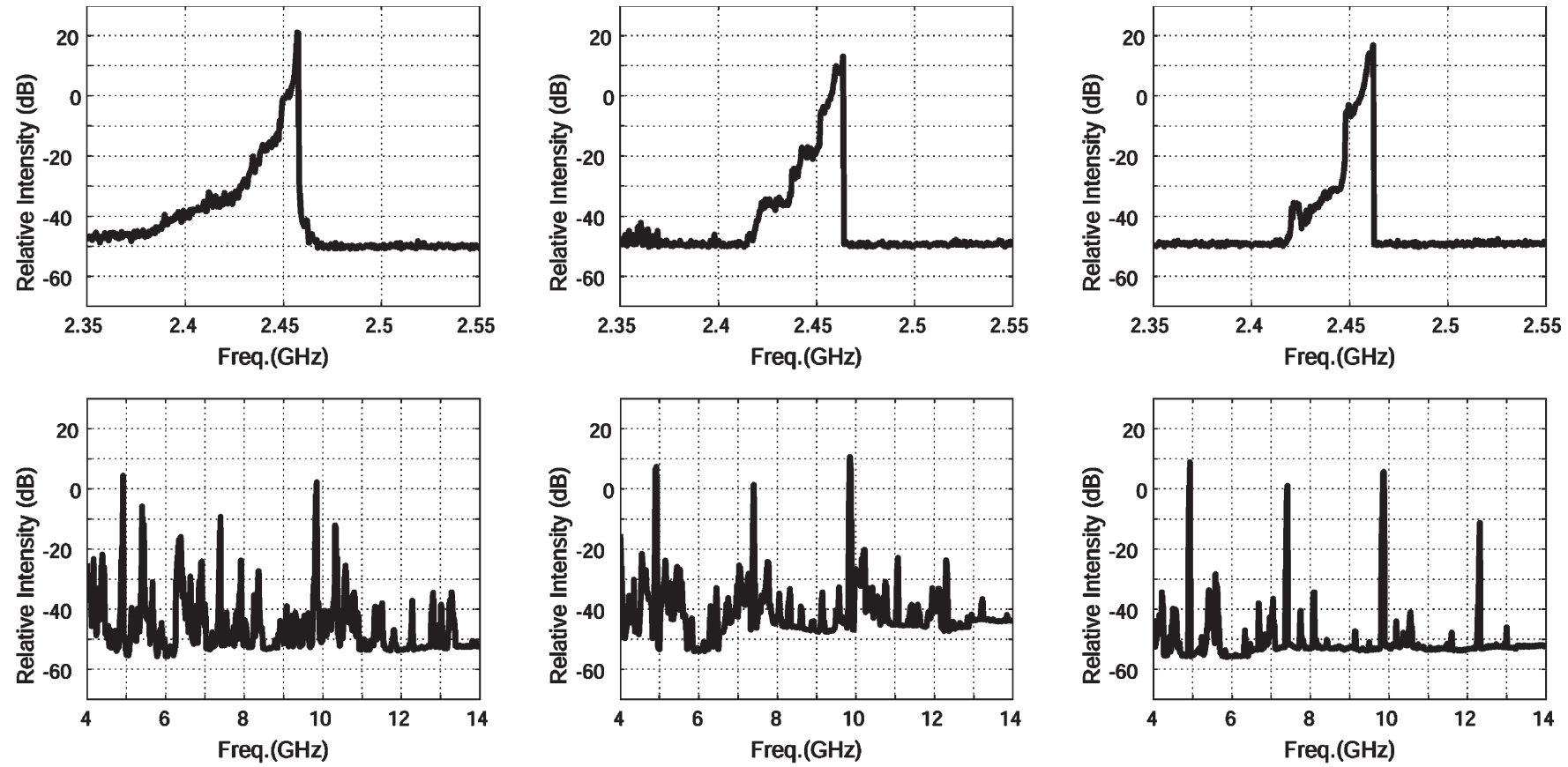

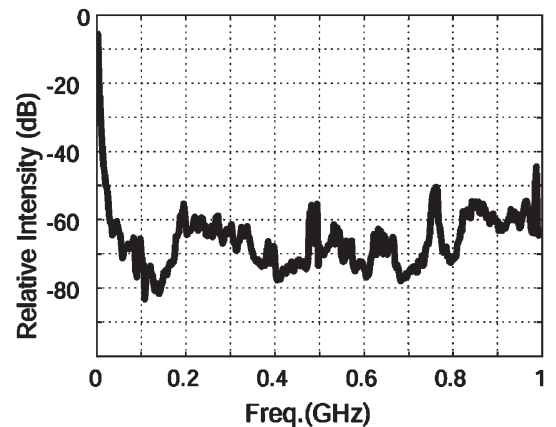

(a)

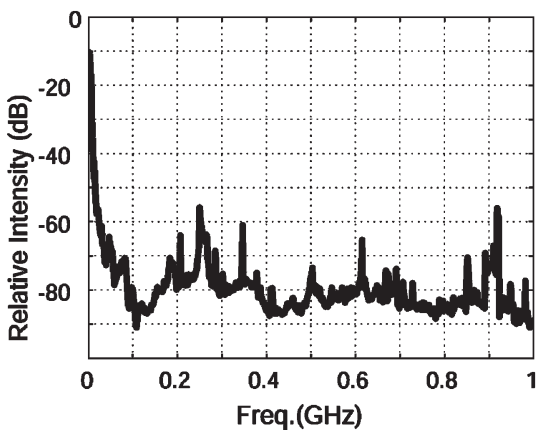

(b)

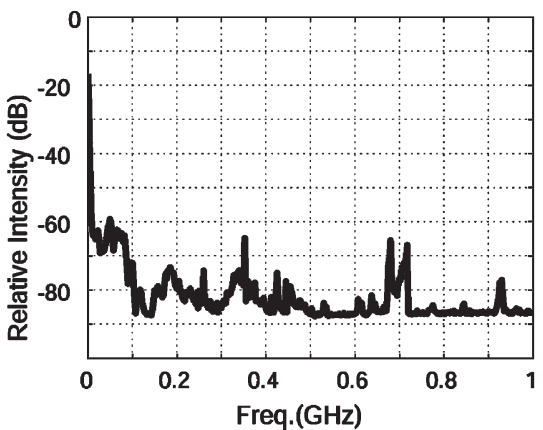

(c)

Fig. 3. Experimental results of the fundamental spectrum (top), high-frequency noise (middle), and line noise (bottom) at $I_{b}=400 \mathrm{~mA}$ and $V_{f}=3.3 \mathrm{~V}$. (a) Conventional magnetron. (b) Magnetron with the cathode shields on both filament ends. (c) Magnetron with a cathode shield on the HV input side.

the magnetron (a) and (b) by about $10 \mathrm{~dB}$. Therefore, shielding the cathode only on the HV input side is more effective in suppressing the sideband noise around the carrier frequency than shielding the cathode on both filament ends.

With regard to the high-frequency spurious noise and the low-frequency line noise, it is obvious that both are drastically reduced by the attachment of the cathode shields on both filament ends and a cathode shield on the HV input side of a magnetron, as shown in the middle row and the bottom row of Fig. 3. Although the cathode shield has a possibility to act simply as a choke in the low frequency bands, the reduction level of the low-frequency line noise from the newly designed magnetron (c) is up to $30 \mathrm{~dB}$, compared to the conventional magnetron (a).

Furthermore, the measurement results clearly specify that both the high-frequency spurious noise and the low-frequency line noise from the magnetron (c) are lower than the magnetron (b). Therefore, a cathode shield on the HV input side is also more effective in suppressing the spurious noise in the high and low frequency bands as well as the fundamental bands than with the cathode shields on both filament ends.

\section{DisCUSSION ON ELECTRON EMISSION From the CATHOde Filament}

The cathode shields play a role in preventing the electron emission from the end portions of the cathode, as Kohsaka et al. mentioned in [13]. Here, we discuss the effects of the cathode shields from the viewpoint of the electron emission. The axial distribution of the filament temperature in a magnetron is measured for discussion.

The axial distribution of the filament temperature was measured with a magnetron whose anode block has an axial slit to see through the cathode filament, as a photo of the magnetron is shown in Fig. 4. The structure of the magnetron is almost the same as a conventional magnetron, except that it has a slit in the anode block. A crystal plate is tightly attached to the slit in order to keep the magnetron tube in vacuum and so that the cathode filament can be directly seen through the crystal plate. The axial distribution of the filament temperature is measured with a disappearing filament optical pyrometer, whose accuracy is within $1 \%$ of the scale range. The spot size was about $0.5 \mathrm{~mm}$, i.e., the filament diameter. The pyrometer was calibrated with a standard lamp. However, the measured temperature could be 


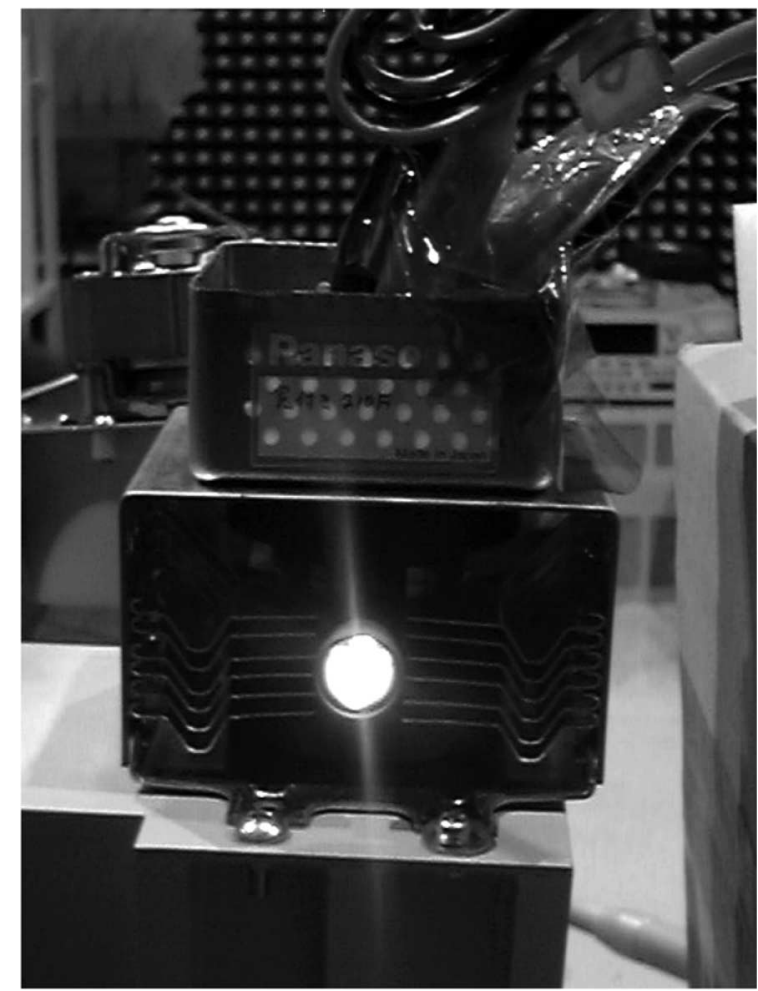

Fig. 4. Photo of the magnetron for the measurements of the filament temperature. The light of the filament is seen through a crystal plate.

slightly lower than the true temperature, as the effect of the crystal plate, which might act as a gray filter, was not calibrated.

First, we describe the axial distribution of the filament temperature in a filament-off operation: The magnetron is operated by a dc-stabilized power supply, and the filament is off. As mentioned in Section I, turning off or cutting back the filament current contributes to the noise reduction of a magnetron. Hence, the axial distribution of the filament temperature in the filament-off operation is valuable in investigating the relationship between the filament temperature and the noise reduction.

Next, we describe the axial distribution of the filament temperature in a half-wave operation: The magnetron is operated by a half-wave voltage doubler, and the filament is on. Then, the effects of the cathode shields are discussed by a comparison between the two sets of the experimental data for the axial distribution of the filament temperature.

\section{A. Axial Distribution of the Filament Temperature in the Filament-off Operation}

Fig. 5 shows the measurement results of the axial distribution of the filament temperature. The horizontal axis shows the axial measurement points on the cathode filament: No. 1 represents the end of the RF output side, No. 4 represents the center of the cathode, and No. 7 represents the end of the HV input side. The solid line with black markers and the dotted line in Fig. 5 represent the curves of the filament temperature in the filament-off operation (the anode current $I_{b}=400 \mathrm{~mA}$ and the filament voltage $\left.V_{f}=0 \mathrm{~V}\right)$ and in the preheating state $\left(I_{b}=\right.$ $0 \mathrm{~A}$ and $V_{f}=3.3 \mathrm{~V}$ ), respectively. Since the oscillation did not continue in the filament-off operation when the magnetron was

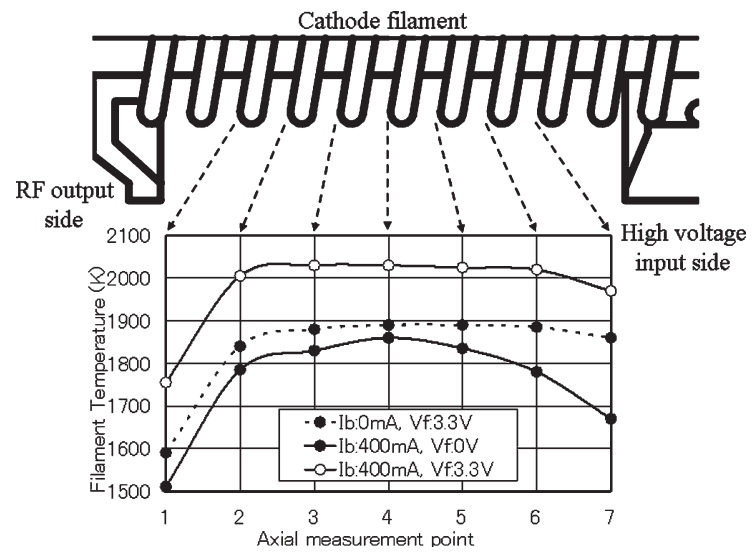

Fig. 5. Axial distributions of the filament temperature. $I_{b}$ : Anode current. $V_{f}$ : Filament voltage. The solid line with black markers, the solid line with white markers, and the dotted line represent the filament temperature in the filament-off operation, in the half-wave operation, and in the preheating state, respectively.

driven by a half-wave voltage doubler, a dc-stabilized power supply was used for the filament-off operation instead.

The experimental results show that the filament in the filament-off operation has a maximum temperature in the center and the filament temperature gradually decreases toward the filament ends. In the filament-off operation, the oscillation continues due to the cathode's self-heating by the back-bombardment energy of the electrons and the secondary electron emission. Hence, the results indicate that the cathode's self-heating is greatest in the center and least at the filament ends.

Furthermore, there is a key difference on the HV input side between the filament-off operation and the preheating state. The difference in the temperature is $200 \mathrm{~K}$ on the $\mathrm{HV}$ input side, whereas it is only $30 \mathrm{~K}$ in the center and $80 \mathrm{~K}$ on the RF output side.

With regard to the filament temperature in the filament-off operation, Brown has mentioned that the temperature becomes the lowest when the thermionically emitting cathode can supply the needed anode current and that this mechanism, the so-called "internal feedback mechanism," contributes to a quiet magnetron operation [14]. Therefore, we assume that the filament on the HV input side looks overheated in the preheating state and that the unnecessary heating by the electric power on the HV input side has some relationship with the noise generation in the filament-"on" operation.

\section{B. Axial Distribution of the Filament Temperature in the Half-Wave Operation}

The solid line with white markers in Fig. 5 shows the measurement results of the axial distribution of the filament temperature in the half-wave operation $\left(I_{b}=400 \mathrm{~mA}\right.$ and $\left.V_{f}=3.3 \mathrm{~V}\right)$.

A key feature is that the axial distribution shows a uniform rise in the temperature by about $150 \mathrm{~K}$, compared to that in the preheating state except at the HV end where the increase is $120 \mathrm{~K}$. This indicates that the cathode's self-heating takes place on both filament ends as well as the center, but is reduced at the $\mathrm{HV}$ end. Thus, the difference of the filament temperature on the 


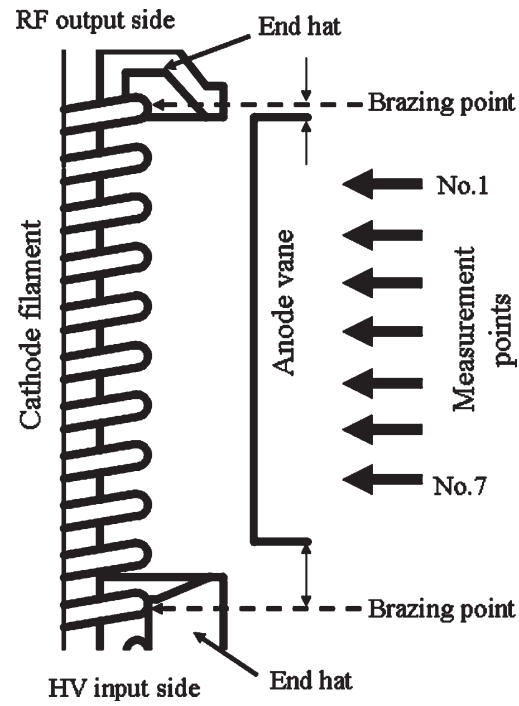

Fig. 6. Brazing points between the cathode filament and the end hats.

$\mathrm{HV}$ input side is $300 \mathrm{~K}$ between the half-wave operation and the filament-off operation.

\section{Causes of Axial Asymmetry of the}

Filament-Temperature Distribution

As shown in Fig. 5, the filament-temperature distribution looks axially asymmetric. This axial asymmetry is partly attributed to both the heat transmission from the filament ends to the end hats and the brazing points between the cathode filament and the end hats.

The cathode filament is brazed to both end hats, and the heat transmission takes place from the filament to the end hats, via the brazing points. Fig. 6 shows the brazing points of a conventional magnetron. When we view the filament from the anode vane as does the optical pyrometer, the axial distance from the brazing point to the nearest measurement point is shorter on the RF output side than on the HV input side. Hence, the filament temperature on the RF output side is measured to be lower than that on the HV input side, since the heat transmission takes place near the measurement points. Finally, the axial distribution of the filament temperature is originally symmetric; however, the measurement results show that the axial distribution looks asymmetric because the center of the cathode filament is out of alignment axially from the center position of the measurement points.

The estimation of the heat conduction from the filament to the end caps is roughly derived from the Fourier's law of heat conduction

$$
Q=-\kappa A \frac{\Delta T}{\Delta x}
$$

where $Q$ is the heat flow per unit time, $A$ is the transversal surface area, $\kappa$ is the conductivity constant, dependent on the nature of the material and its temperature, $\Delta T$ is the temperature difference, through which the heat is transferred, and $\Delta x$ is the material length. $\Delta T$ is given by the temperature difference in the preheating state, which is shown as the dotted line in Fig. 5, between the measurement point No. 1 and No. 2: $\Delta T$ is
TABLE II

RATIOS OF THE CURRENT DENSITY NORMALIZED BY THE RF OUtPUT SIDE IN THE FILAMENT-OFF OPERATION. (HVD STANDS FOR HALF-WAVE Voltage DoubleR

\begin{tabular}{|c||c|c|}
\hline & $\begin{array}{c}\text { Filament-off } \\
\text { operation }\end{array}$ & $\begin{array}{c}\text { Operation } \\
\text { by a HVD }\end{array}$ \\
\hline \hline RF output side & 1 & $1.5 \times 10$ \\
\hline Center & $3.5 \times 10$ & $1.3 \times 10^{2}$ \\
\hline HV input side & 6.1 & $8.5 \times 10$ \\
\hline
\end{tabular}

$-280 \mathrm{~K} . A$ is the surface area of the filament: $2.0 \times 10^{-7} \mathrm{~m}^{2}$, and $\Delta x$ is the filament length per turn: $1.3 \times 10^{-2} \mathrm{~m}$ for the measured magnetron. Substituting the conductivity of tungsten at a temperature of $1000 \mathrm{~K}: 1.2 \times 10^{2} \mathrm{~W} / \mathrm{m} / \mathrm{K}$ for $\kappa, Q$ is calculated to be $0.52 \mathrm{~W}$. The ohmic dissipation per turn is given by the filament voltage $(3.3 \mathrm{~V}) \times$ the filament current $(10.5 \mathrm{~A}) / 10$ turns for the measured magnetrons: $3.5 \mathrm{~W} / \mathrm{turn}$. Therefore, heat conduction to the end caps accounts for $15 \%$ of the ohmic dissipation per turn.

Note that, the axial position of the anode vanes is also off the center to the RF output side in a conventional magnetron. Therefore, the larger number of the electrons will flow in the anode on the HV input side, compared to the RF output side.

\section{Comparison of the Thermionic Emission}

Now, we discuss the thermionic emission from the cathode filament by the Richardson-Dushman's equation, which relates to the thermionic current-density limit and the temperature of the emitting material:

$$
J=A T_{\mathrm{K}}^{2} \exp \left(-e \phi / k T_{\mathrm{K}}\right)
$$

where $J$ is the thermionic current-density limit, $e$ is the electron charge, $k$ is the Boltzmann constant, $A$ is the thermionicemission constant, $T_{K}$ is the temperature in Kelvin, and $\phi$ is the work function of the emitting material (e.g., [15]). For a microwave oven magnetron, the emitting material of the filament is carburized thoriated tungsten $\left(\mathrm{W}_{2} \mathrm{C}+\mathrm{Th}\right)$, and its work function is $2.18 \mathrm{eV}$ [16].

From the experimental data, the filament temperatures on the RF output side (measurement point No. 1), in the center (No. 4), and on the HV input side (No. 7) were 1510, 1860, and $1670 \mathrm{~K}$ in the filament-off operation, and 1760, 2030, and $1970 \mathrm{~K}$ in the half-wave operation, respectively. Table II shows the ratios of the thermionic current-density limit, which are derived from (2), normalized by the temperature on the RF output side in the filament-off operation. From Table II, the thermionic current-density limits on both of the filament ends in the half-wave operation are 14-15 times larger than those in the filament-off operation. Moreover, the thermionic currentdensity limit on the HV input side is 5.7 times larger than that on the RF input side in the half-wave operation.

\section{E. Effects of the Cathode Shields for the Spurious-Noise Reduction}

As mentioned in Section IV-A, the unnecessary heating by the electric power on the HV input side could have some 
relationship with the noise generation from a magnetron in the filament-"on" operation. From the experimental results in Fig. 3, a cathode shield on the HV input side greatly suppresses the spurious noise. Thus, a cathode shield, which prevents the electrons from going directly into the interaction space, contributes to the spurious-noise reduction, as if it makes an axial distribution of the filament temperature similar to that in the filament-off operation virtually.

Finally, we conclude that the attachment of the cathode shield on the HV input side is found to be highly effective for the spurious-noise reduction, by preventing the electron emission from there.

\section{Discussion on Noise-Reduction Mechanisms}

Now, we discuss the noise-reduction mechanisms that are done by the attachment of the cathode shields.

\section{A. Sideband Noise in the Fundamental Bands}

First, we describe a noise-reduction mechanism in the fundamental bands.

The output frequency of a magnetron is determined by the loaded anode structure, and the electron spokes are synchronized with the RF field. Then, the $N / 2$ electron spokes are formed in the $\pi$-mode operation of an $N$-cavity magnetron. Meanwhile, the azimuthal mean velocity of the electrons $\langle v(\theta)\rangle$ is determined by the mean $\boldsymbol{E} \times \boldsymbol{B}$ drift velocity, i.e., $\langle v(\theta)\rangle=$ $\left\langle E_{r}\right\rangle / B_{z}$, where $\left\langle E_{r}\right\rangle$ is the radial mean electric field and $B_{z}$ is the axial magnetic field. When the $\pi$-mode frequency is pulled by the loaded anode, $\langle v(\theta)\rangle$ is also tuned by changing $\left\langle E_{r}\right\rangle$. Therefore, the circulating frequency of the electron spokes $f$ can be roughly expressed as follows:

$$
f=\frac{N}{2} \frac{|\langle v(\theta)\rangle|}{2 \pi r_{\mathrm{a}}}=\frac{N}{2} \frac{1}{2 \pi r_{\mathrm{a}}} \frac{\left|\left\langle E_{r}\right\rangle\right|}{B_{z}}
$$

where $r_{\mathrm{a}}$ is the anode radius.

From the time-domain measurements of the magnetron noise in the fundamental frequency bands [17], the sideband noises below $2.45 \mathrm{GHz}$ are generated at the startup and in a low anode-current region, when the electron spokes are about to be formed and synchronized with the RF field. Hence, the circulating frequency of the electrons in the interaction space at these stages is also expressed by (3).

Now, we consider the azimuthal drift motions of the electrons on both sides of the filament ends. A traditional twodimensional (2-D) analysis of a magnetron has treated the magnetic-flux density as a constant in the axial direction. In a practical magnetron, however, the nearer the magnetic pole, the larger the magnetic-flux density. In the case of the conventional magnetron, the axial magnetic-flux density $B$ in the vicinity of the anode vanes on the filament ends increases by a few percent, compared to the center. Hence, the circulating frequency $f$ on the filament ends becomes lower than that at the center, assuming $\left|\left\langle E_{r}\right\rangle\right|$ is uniform in the axial direction.

Furthermore, the magnetron with a cathode shield on the HV input side suppresses the spurious noise in the fundamental bands up to $2.45 \mathrm{GHz}$, compared to the magnetron with the cathode shields on both filament ends. This noise reduction takes place because the cathode shield of the magnetron (c) extends toward the center of the filament more than the magnetron (b), as shown in Fig. 1. In the magnetron (c), the cathode shield affects the azimuthal $\boldsymbol{E} \times \boldsymbol{B}$ drift motion of the electrons near the center, so that the spurious noise near the peak frequency are reduced.

Finally, we conclude that a cause of the noise reduction, owing to the cathode shields, is attributed to the disappearance of the azimuthal $\boldsymbol{E} \times \boldsymbol{B}$ drift motion of the electrons on the filament ends. Especially, from the axial distribution of the filament temperature shown in Fig. 5, a cathode shield on the HV input side is much more effective in reducing the spurious noise, because more electrons are shielded.

\section{B. Spurious Noise in High and Low Frequency Bands}

With regard to the spurious noise in the high and low frequency bands, oscillations by the circulating electrons [9] in a magnetron are considered as one of the noise-generation mechanisms.

The oscillations by the circulating electrons are derived from the Hartree's condition [18] as follows:

$$
f_{n}=\frac{e n B_{z}}{4 m \pi} \frac{r_{\mathrm{a}}^{2}-r_{\mathrm{c}}^{2}}{r_{\mathrm{a}}^{2}}\left\{1-\sqrt{1-\frac{8 m V}{e B_{z}^{2}} \frac{r_{\mathrm{a}}^{2}}{\left(r_{\mathrm{a}}^{2}-r_{\mathrm{c}}^{2}\right)^{2}}}\right\}
$$

where $f_{n}$ is the oscillating frequency, $n=0,1,2, \ldots, N / 2, V$ is the anode voltage, $r_{\mathrm{a}}$ and $r_{\mathrm{c}}$ are the radii of the anode and the cathode, $B_{z}$ is the magnetic field, $m$ is the electron mass, and $e$ is the electron charge.

As $f_{n}$ is a function of $B_{z}$, the bandwidths of these oscillating frequencies by the circulating electrons broaden due to the axial variation of the magnetic-flux density. In the same manner, the harmonics in the high frequency bands also broaden. Thus, the cathode shields on both of the filament ends, especially on the HV input side, are also effective in suppressing the spurious noise in the high and low frequency bands.

Note that, the $\pi-1$ mode at a frequency of about $4.4 \mathrm{GHz}$ is suppressed up to $20 \mathrm{~dB}$ by the cathode shield on the HV input side. Although the cause has not been found yet, the cathode shield also works for the suppression of the $\pi-1$ mode or the separation between the $\pi$ mode and the $\pi-1$ mode.

\section{CONCLUSION}

A magnetron with a cathode shield on the HV input side was newly designed, and its spurious noise was measured. From the experimental results, we conclude that a cathode shield on the HV input side works quite effectively in suppressing the spurious noise when it is driven by a half-wave voltage doubler.

A magnetron is commonly operated by a half-wave voltage doubler or a full-wave rectified power supply in an oven, so that it alternately oscillates and breaks during its operation. Then, the filament-off technique has a difficulty in maintaining the magnetron operation due to the excessive falloff of the filament 
temperature at the "break" stage. Therefore, our newly designed magnetron has a great deal of potential for the development of a low-noise magnetron without turning off or cutting back the filament current.

It is of great interest that the "axially asymmetric" configuration of the cathode shield works more than the "axially symmetric" configuration and that the relation between "axially asymmetric" and "axially symmetric" has also been observed in an experimental study on the "magnetic priming" [11], which reported that the "axially asymmetric magnetic priming" has been more effective for the noise and startup of the magnetrons than the "axially symmetric magnetic priming." Therefore, a study on these "axially asymmetric" issues will be important to figure out the generation mechanisms of the magnetron noise.

It will also be necessary to analyze a magnetron from a 3-D perspective for the development of a low-noise magnetron. For example, the axial electrostatic field $E_{z}$ begins to emerge on the filament ends due to the existence of the end hats and the cathode shields. Or, the magnetic fields around the filament ends are not perfectly parallel to the axial direction, and the radial magnetic field component $B_{r}$ slightly exists there. Thus, the motions of the electrons or the electromagnetic fields on the filament ends are complex because of not only the dominant $\left(E_{r}, B_{z}\right)$ components but also the slight $\left(E_{z}, B_{r}\right)$ components. Since it is too complicated to solve these motions analytically, the 3-D computer simulations will be a powerful tool for solving these problems.

\section{ACKNOWLEDGMENT}

Experimental measurements have been conducted in the anechoic radio wave chamber of the "Microwave Energy Transmission Laboratory (METLAB)," which is one of the facilities of the cooperative study programs of the Research Institute of Sustainable Humanosphere (RISH), Kyoto University.

\section{REFERENCES}

[1] P. E. Glaser, F. P. Davidson, and K. Csigi, Solar Power Satellites. Chichester, U.K.: Wiley, 1998.

[2] W. C. Brown, "Beamed microwave power transmission and its application to space," IEEE Trans. Microw. Theory Tech., vol. 40, no. 6, pp. 12391250, Jun. 1992

[3] T. Mitani, N. Shinohara, H. Matsumoto, and K. Hashimoto, "Experimental study on oscillation characteristics of magnetron after turning off filament current," Electron. Commun. Jpn. Part II. Electron., vol. 86, no. 5, pp. 1-9, May 2003.

[4] _ " "Improvement of spurious noises generated from magnetrons driven by DC power supply after turning off filament current," IEICE Trans. Electron., vol. E86-C, no. 8, pp. 1556-1563, Aug. 2003.

[5] W. C. Brown, "Update on the solar power satellite transmitter design," Space Power, vol. 6, no. 2, pp. 123-135, 1986.

[6] H. Matsumoto, "Research on solar power station and microwave power transmission in Japan: Review and perspectives," IEEE Microw. Mag., vol. 3, no. 4, pp. 36-45, Dec. 2002.

[7] I. Tahir, A. Dexter, and R. Carter, "Noise performance of frequency-and phase-locked CW magnetrons operated as current-controlled oscillators," IEEE Trans. Electron Devices, vol. 52, no. 9, pp. 2096-2103, Sep. 2005.

[8] N. Shinohara, T. Mitani, and H. Matsumoto, "Development of phase and amplitude controlled magnetron," in Proc. 6th IVEC, Apr. 2005, pp. 61-64.

[9] K. Yamamoto, H. Kuronuma, T. Koinuma, and N. Tashiro, "A study of magnetron noise," IEEE Trans. Electron Devices, vol. ED-34, no. 5, pp. 1223-1226, May 1987.

[10] V. B. Neculaes, R. M. Gilgenbach, Y. Y. Lau, M. C. Jones, and W. M. White, "Low-noise microwave oven magnetrons with fast start- oscillation by azimuthally varying axial magnetic fields," IEEE Trans. Plasma Sci., vol. 32, no. 3, pp. 1152-1159, Jun. 2004.

[11] V. B. Neculaes, M. C. Jones, R. M. Gilgenbach, Y. Y. Lau, J. W. Luginsland, B. W. Hoff, W. M. White, N. M. Jordan, P. Pengvanich, Y. Hidaka, and H. L. Bosman, "Magnetic perturbation effects on noise and startup in DC-operating oven magnetrons," IEEE Trans. Electron Devices, vol. 52, no. 5, pp. 864-871, May 2005.

[12] J. I. Kim, J. H. Won, and G. S. Park, "Electron pre-bunching in microwave magnetron by electric priming using anode shape modification," in Proc. 6th IVEC, Noordwijk, The Netherlands, Apr. 2005, pp. 55-56.

[13] A. Kohsaka, H. Saitoh, and T. Kawaguchi, "Low profile and clean spectrum magnetron," J. Microw. Power Electromagn. Energy, vol. 24, no. 1, pp. 3-13, 1989.

[14] W. C. Brown, "The SPS transmitter designed around the magnetron directional amplifier," Space Power, vol. 7, no. 1, pp. 37-49, 1988.

[15] J. A. S. Gilmour, Microwave Tubes. Norwood, MA: Artech House, 1986, ch. 5 .

[16] J. Ishikawa, Science and Technology of Charged Particle Beams (in Japanese). Tokyo, Japan: Corona Publishing Co., Ltd., 2001.

[17] T. Mitani, N. Shinohara, H. Matsumoto, M. Aiga, N. Kuwahara, and T. Handa, "Time domain analysis of noises generated from microwave oven magnetron," Electron. Commun. Jpn. Part II. Electron., vol. 88, no. 10 , pp. 28-36, Oct. 2005.

[18] J. C. Slater, Microwave Electronics. New York: Van Nostrand, 1950, ch. 13.

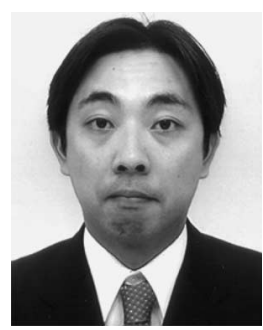

Tomohiko Mitani (M'05) received the B.E. degree in electrical and electronic engineering, the M.E. degree in informatics, and the Ph.D. degree in electrical engineering from Kyoto University, Kyoto, Japan, in 1999, 2001, and 2006, respectively.

He was an Assistant Professor with the Radio Science Center for Space and Atmosphere, Kyoto University, in 2003. He has been an Assistant Professor with the Research Institute for Sustainable Humanosphere, Kyoto University, since 2004. His current research interests include the experimental study on magnetrons, microwave power-transmission systems, and applied microwave engineering.

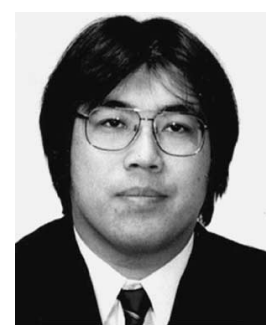

Naoki Shinohara (M'06) received the B.E. degree in electronic engineering and the M.E. and Ph.D. (Eng.) degrees in electrical engineering from Kyoto University, Kyoto, Japan, in 1991, 1993, and 1998, respectively.

He was a Research Associate with the Radio Atmospheric Science Center, Kyoto University, in 1998 and with the Radio Science Center for Space and Atmosphere, Kyoto University in 2000, where he became an Associate Professor in 2001. He has been an Associate Professor with the Research Institute for Sustainable Humanosphere, Kyoto University, since 2004. He has been engaged in the research on the solar-power station/satellite and microwave power-transmission system. 


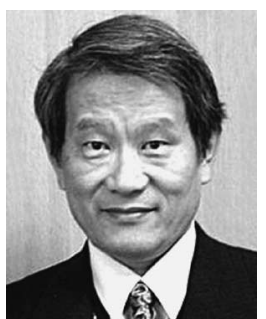

Hiroshi Matsumoto (SM'96-F'03) received the B.E. degree in electronic engineering, and the M.E. and Ph.D. (Eng.) degrees in electrical engineering from Kyoto University, Kyoto, Japan, in 1965, 1967, and 1973, respectively.

He was a Professor with the Radio Atmospheric Science Center, Kyoto University in 1977, where he was the Director from 1992 to 1998 . He was a Professor with the Radio Science Center for Space and Atmosphere, Kyoto University in 2000, where he was the Director from 2002 to 2004. He was a Professor with the Research Institute for Sustainable Humanosphere, Kyoto University from 2004 to 2006, where he was the Director from 2004 to 2005. He has been an Executive Director with Kyoto University since 2005. He has had appointments as a Visiting Professor with Institute of Space and Astronautical Science, Japan Aerospace Exploration Agency, as a Guest Researcher and Advisory Board Member with Communications Research Laboratory, Japan, and as a Guest Professor with the Wuhan University, China. He has been engaged in the research on space plasma physics, space radio science and engineering, solar-power station/satellite, and microwave power-transmission system.

Dr. Matsumoto received the Tanaka Date Award from the Society of Terrestrial Magnetism and Electricity of Japan in 1975, the NASA Group Achievement Award in 1993 and 1998, the American Geophysical Union (AGU) Fellow Award in 1999, the Sida-Rinzaburo Award in 1999, and the ISAP2000 Paper Award in 2000. He received the President Award of Kansai Bureau of Telecommunications in 2004. He is an RAS-Associate of the Royal Astronomical Society, United Kingdom, in 2004. He is a member of the AGU and a member of the Society of Geomagnetism and Earth, Planetary and Space Sciences (SGEPSS). He was the President of the International Union of Radio Science (URSI) from 1999 to 2002.

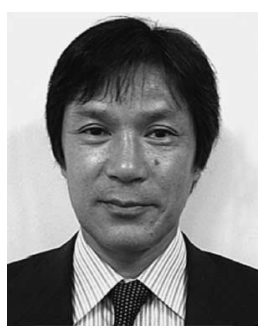

Masayuki Aiga graduated from Electrical Engineering Department, Owase Industrial High School in 1970.

He was with the Matsushita Electronic Instruments Corp. from 1999 to 2005. He has been with the Panasonic Semiconductor Discrete Devices Company, Ltd., since the merger of the companies. He has been engaged in the development of magnetrons.

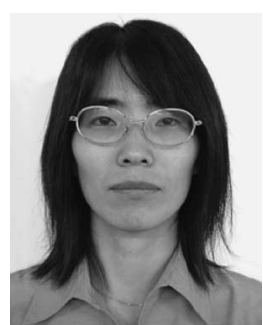

Nagisa Kuwahara received the B.E. degree in electronic engineering from Utsunomiya University in 1989.

She was with the Matsushita Electronic Instruments Corp. from 1989 to 2005. She has been with the Panasonic Semiconductor Discrete Devices Company, Ltd., since the merger of the companies. She has been engaged in the development of magnetrons.

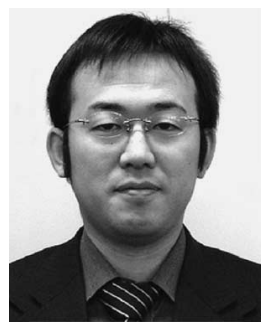

Takeshi Ishii received the B.E. degree in electronic engineering from Ashikaga Institute of Technology in 1998.

He was with the Matsushita Electronic Instruments Corp. from 1998 to 2005. He has been with the Panasonic Semiconductor Discrete Devices Company, Ltd., since the merger of the companies. He has been engaged in the development of magnetrons. 